\title{
Research on the Style Characteristics of Network Hosts
}

\author{
-- Taking Wang Nima's Style Characteristics as An Example
}

\author{
Xinhua Li \\ School of Literature and Journalism and Communication, Shandong University of Technology, Zibo, Shandong, China
}

\begin{abstract}
There is a distinct difference between the network host style and the traditional host style. Wang Nima, as the "most positive host of three views of speech", is very successful in thinking from the perspective of social psychology. Wang Nima has become one of the symbols of the violent image subculture group through the styles of hiding identity, symbolized cartoon image, symbolized audio language and paralanguage expression, phonetic vocabulary grammar deviation and so on. The use of Wang Nima's image can better stimulate the audience to communicate and deliver the program. At the same time, the youth subculture audience speaks and spits with "opinion leaders" such as Wang Nima, and "opinion leaders" such as Wang Nima become spokespersons with obvious group characteristics. However, its disadvantage is that it can influence the public opinion trend of news on the Internet, and further interfere with the consciousness of individual thinking in the group.
\end{abstract}

Keywords: Network host style, Hide identity, Symbolized cartoon image, Symbolic language, Deputy language, Nonsense language, Rage Comic image.

\section{Introduction}

High-quality online content circle powder a large number of new generation online audience, many network phenomenon IP has a large number of fans. Fans hold groups online and strengthen their sense of belonging to the subcultural circle in the interaction. For example, Wang Nima, the host of "Runaway big event", didn't give him a real identity, but hosted it through symbolic cartoon characters. As the host of an online talk show, we use concrete examples to make an analogy. The image appearing in the show is similar to the purring in the children's channel Wisdom Tree, and the idea of communication is similar to the virtual host Blue Bear waldo in the third episode of the second season of the British drama Black mirror. Host Wang Nima, as a runaway team, successfully created "opinion leader" with very strong secondary attributes. As "opinion leaders", guiding public opinion needs to conform to the universal values and moral standards of the masses.

Host Wang Nima has always been praised by his fans as "the most correct host with three views of speech", which is in line with the communication purpose and economic purpose of the runaway team. It is very successful to choose the cartoon image of Rage Comic as the image of the host Wang Nima from the perspective of social psychology. As one of the symbols of the subculture group of Rage Comic image, using the image of Wang Nima can better stimulate the audience to communicate and transmit programs. At the same time, the audience of youth subculture speaks and spits out with "opinion leaders" such as Wang Nima, and "opinion leaders" such as Wang Nima become spokespersons with obvious group characteristics. However, its disadvantage is that it can influence the public opinion trend of news on the Internet, and further interfere with the consciousness of individual thinking in the group.

\section{Viewing the Style of Network Host from Wang Nima}

\subsection{Wang Nima's True Identity Guess}

Wang Nima, according to the information provided by the runaway employee, is Ren Jian, CEO of Xi 'an Momo Information Technology Co., Ltd., born on January 8th, 1990, and graduated from LSE, majoring in social psychology. On September 6th, 2010, he established Xi 'an Momo Information Technology Co., Ltd., and in the same year, he produced runaway comics and became the official editor. In 2013, the news network talk show "Runaway big event" was produced by Ren Jian company team. In the first program, Ren Jian, CEO, wore silk stockings and a thick headgear symbolizing "runaway" culture, and began to host the showplaying the host "Wang Nima". From the first season of "Runaway big event" broadcast in 2013 to the end of the fifth season of this year, the host Wang Nima never took off his sleeve. In the past five years, the runaway official has never released the real information of the host. For the sake of rigor, the above descriptions of Ren Jian are all true, but as the screen image of Wang Nima, as the audience, no one knows the true appearance under the hood, whether the runaway team has changed the host in the past five years, and whether Wang Nima himself is still Ren Jian, which involves trade secrets, so I can't make an accurate conclusion. What is certain is that Wang Nima is a successful work created by Ren Jian and his company team, which does not affect the author's analysis of the style characteristics hosted by Wang Nima.

\subsection{Wang Nima's Symbolic Cartoon Image}

\subsubsection{Wang Nima's Symbolic Cartoon Image}

"At the beginning of the program production," Runaway big event "targeted audiences from 3 to 29 years old." (Excerpted from the speech delivered by Ren Jian, the founder of Outbreak, at the 4th ORZ Study Abroad Elite Festival of New Oriental on June 24th, 2017) Wang Nima's basic image is "jeans with a distinctive T-shirt printed with the 
head of Outbreak", "a bag of toilet paper at the waist", "a chubby body with a big belly breast", and the most obvious image feature is that he wears a "Outbreak" hood on his head. Pluralistic symbolic features constitute Wang Nima's image style features, especially the headgear, which not only effectively blocks his face, but also the shocking expression presented by the headgear can not only clearly reflect the radical connotation of the program, but also provide the best evidence for many ironic narrations in the program materials. The symbolic cartoon image further weakens people's exploration of Wang Nima's true identity, but focuses on Wang Nima itself.

\subsubsection{Wang Nima's Symbolic Cartoon Image Has the Characteristics of Youth Subculture}

Wang Nima's image is derived from emoticons created in "runaway comics". At first, its company was used for serializing comics, social expressions and other functions. By matching expression templates and expressing words, exaggerated or even distorted cartoon expressions were used to describe the network and the real world. Satire and venting teenagers' dissatisfaction with social problems are the manifestations of the transformation of social contradictions in the mainland at the present stage in the field of youth subculture. The users are mainly post-90s and post-00s. After the broadcast of the online talk show "Runaway big event", the host Wang Nima appeared as a runaway comic protagonist, which deepened the style characteristics of the youth subculture in Wang Nima's image.

Wang Nima's playful expression of "running away from the headgear" represents the rebellious gene in the youth subculture, and the toilet paper on the shoulder inclined across suggests that Wang Nima launches a large-scale public welfare activity in September for teenagers every year.

\subsection{Wang Nima's Symbolic Voiced Speech and Paralanguage Expression}

\subsubsection{Symbolic Voice Expression}

During the first to fifth season of Runaway big event, the host Wang Nima showed many symbolic audio language expressions, with conversational style as the expression form. For example, the opening words "Woo-la-la-la-la-la" and the ending words "Jing Ke pricks the king of Qin" of each period become important symbols of audio language in their programs.

As we all know, audio language has stronger, deeper and more direct sensibility than written language. "Woo-la-la-lala-la", analyzed from the natural language level, is only a mood word when Wang Nima presides over the appearance prefix. As an audience familiar with the subculture background of Runaway big event, when we understand this modal particle at the logical language level, we give it new semantics, and it becomes "dirty la la la la la la".

"Dirty", as a vocabulary category of youth subculture popular on the Internet, has a new meaning. "Dirty", as an online word with funny and playful nature, is widely popular on the Internet in recent years and belongs to the category of online popular culture. Words or things that are meant to make people feel embarrassed, lustful, shy and bad are mostly used to obscure things or languages that are easy to arouse people's lust or interest reverie. " (Baidu entry modern network terminology interpretation)

In the traditional culture of the mainland, "sex" changes color, and students lack the popularization of sex education in their families, schools and society. In the traditional culture, education in the mainland has long suppressed and rejected anything related to sex and voiced language and words to express its contents. Wang Nima uses symbolic audio language to express, and uses "dirty la la la la la la" to effectively satirize and fight back the feudal conservative culture. "La la la la la la la" after "dirty" is an emotional expression of human liberation.

"Jing Ke pricks Qin Wang", as the symbolic ending of the program, has also been endowed with many connotations. "At 23 o'clock on September 22nd, 2012, rage comic officially released Weibo: Jing Ke pricks Qin Wang, ask for the next sentence! One of them replied: Two hairy legs on his shoulders. Forwarded by rage comic official Weibo. " (Collected from Sina Weibo) It is not difficult to see that there is vulgar taste in it. After the first season of Runaway big event started, the host Wang Nima used this "vulgar meaning" password at the end of the program. As an online news talk show, "Runaway big event" has great influence and appeal among teenagers. After reporting on child abuse incidents in Yang Yongxin and Yuzhang Academy in the fifth quarter of 2017, "Runaway big event" was removed from the whole network for rectification. The 36th program was deleted from the whole network, and the comments on talk shows involving sensitive current political news in the past were also deleted extensively. "Jing Ke pricks Qin Wang", as the ending of this program, is also gradually interpreted by some Rage Comic fans as "fighting against authority", and this statement has also received a response from the audience.

The audience's interpretation of symbolic audio language has gradually deviated from the original meaning of the program, and the audience has begun to actively participate in the processing and dissemination of information from the passive acceptance of information transmitted by the media. The audience is no longer the "rabble" who only passively received information in the pre-Internet era. This is actually a very meaningful reflection brought by the online talk show Runaway big event.

\subsubsection{Symbolic Paralinguistic Expression}

Wang Nima has a fixed "runaway" style headgear. In the facial expression of the headgear, the paralanguage is an exaggerated and playful expression, and the playful emotion expressed by the paralanguage is also very consistent with the skills used by Wang Nima in vocal language expression. This symbolic paralanguage expression further strengthens Wang Nima's unique style feature in talk show hosts-Nima humor, and at the same time reflects the rebellious genes in youth culture, and further draws closer to the adolescent audience.

Wang Nima, a roll of toilet paper diagonally across the waist for each program. From the perspective of symbolic paralanguage expression, this kind of costume language design is very successful. Toilet paper is a metaphor for "diaosi". Most of the audiences watching Wang Nima talk show are "grassroots groups". Its purpose is obvious, and it is a self-deprecating effect closer to the mainstream audience. In short, symbolic audio language and paralanguage distributed in the program are one of the distinctive style features of host Wang Nima.

\subsection{Nonsense Language Expression Style}

\subsubsection{The Comic Language Expression of Nonsense Shows the "Tragic" Core of the Current Society}

There are many funny "Wulitou" features in Wang Nima's audio language expression, and "Wulitou" humor is a major feature of Stephen Chow comedies. "'Wulitou' originated 
from the dialect of Shunde District, Foshan City, Guangdong Province. In the folk dialect, the original meaning is swearing, which means that nothing one can do is useless." (Qian Wei. Wulitou Movies and Folk Culture in Stephen Chow. Journal of Hainan Normal University. 2013) The language or behavior of "Wulitou" actually has profound social connotation and touches the essence of things in a playful way.

As a news talk show, "Runaway big event" hosted by Wang Nima is bound to involve many news hotspots. It is inevitable to grasp the scale when making news comments to avoid the program touching politically sensitive issues and being blocked. The language expression style of "Wulitou" provides a breakthrough for the program to criticize such social problems.

In May, 2011, the "Five Streets and Teenagers" incident made this normally young pioneers team logo, which triggered a nationwide discussion. Then, in the first season, Runaway big event produced a special program to satirize the government-based family education concept of social deformity behind the incident of Huang Yibo, the deputy captain of the 13-year-old youth team of "Five Streets", interspersed with the talk show in the form of the comedy short film "Crime-breaking Primary School", in which the captain of the Five Streets is old-fashioned, judging the political ecology of the young pioneers in today's campus environment as a young politician, pointing out the country and being knowledgeable. The program vilifies and subverts all aspects of the image characteristics such as the thought, appearance, age and so on, and deconstructs the dual identities of the primary school students and the vice-captain of the Young Pioneers with nonsense comedy language.

Official-oriented evaluation of social status by official titles is undoubtedly the dross left over from feudal culture. In modern family education, official-oriented thought poisons the mental health of teenagers. Wang Nima shows the core of "tragedy" under social problems through the nonsense comedy language style of the program, such as magnifying glass, arouses the audience's thinking, shows the different forms of expression of news talk shows, and gives a new artistic height to the style characteristics of network talk show hosts.

\subsubsection{The Concrete Presentation of Wang Nima's Nonsense Language Expression Style in the Program}

"Wulitou language style is characterized by the deviation of language world. Common deviations mainly include phonetic deviation, lexical deviation and grammatical deviation." (Wu Ying, Yu Bin. Analysis of Language Deviation of "Wulitou" _ Taking Stephen Chow films as an example)

(1) Phonetic deviation

In "Runaway big event", Wang Nima uses nonsense language to express the characteristics of phonetic deviation in style, which is obvious to England, and it is called "Ying gay Lan" for many times. The homophonic way is a metaphor for the prosperity of same-sex culture in Britain.

Wang Nima skillfully rhymes in commenting on news to strengthen the effect of nonsense language expression. Ex.: I suddenly returned to my hometown at night, and forced the demolition team, which has been demolished! Which one is stronger in English, Shaolin Temple, find Li Yang! Rhyme antithesis comments strengthen the humorous and nonsense style image of Wang Nima host.

(2) Lexical deviation
In the hosting process of Nima, the popular online expressions and English were mixed and created, and a new sentence "Why are you so diao?" was created in the third season of Runaway big event by the method of sharing English and Chinese. ! ", meaning "why are you so diao? !" Used to satirize shameful, illegal and criminal news events or news figures.

Wang Nima also refitted and innovated a large number of everyday sayings during the hosting process. For example, in the news about old people touching porcelain, Wang Nima coined the word "Xiong Laoren" in order to better express his dissatisfaction with these old people's behavior without causing personal harm to these old people. This word is easily reminiscent of the word "Xiong Haizi". "Xiong Haizi" refers to those minors who do bad things, are naughty or violate social order and good customs. The "Xiong Laoren" here naturally misappropriates its semantics, but at the same time it also adds a layer of semantics: children do this because they are still in the stage of developing values, while the elderly do this for the embodiment of disrespect for the elderly, and the irony is prominent.

(3) Grammatical deviation

In the process of Wang Nima's hosting, he cleverly mocked news events by stealing or obscuring the subject. For example, in 2018, the national football team defeated Wales 0-6, setting the biggest losing record at home. Wang Nima said, "There are two kinds of football matches in the world that are the most boring, because you already know the result without looking at them. One is Chinese men's soccer, and the other is Chinese table tennis. The former can't beat anyone, and the latter can't be beaten by anyone. " Chinese men's soccer team has been losing, while Chinese table tennis team has been winning.

Determine the production and consumption objects and consumption patterns. From the very beginning, the works produced by online content determine the age characteristics, education level, spending power and many other factors of the consumers. At present, the great development of mobile payment is also changing the way of making profits and consuming online content. The talk show "Luo Ji Thinking" is the beginning of the transformation from internet advertising profit to content payment.

The success of the host can not be separated from the packaging and improvement of the team, and successful audio language works should conform to the cultural connotation of the audience. Wang Nima's nonsense language expression style has been carefully designed and planned. The innovation of Wang Nima's team for China's online video programs, especially talk shows, has further promoted the liveliness of the online market.

\section{References}

[1] Shi Yanzi. The role of network host in the self-media era [J]. News lovers: October 2016.

[2] Cheng Yanlin. The development strategy of broadcasting and hosting in the self-media era [J]. The World of Sound Screen: August 2017.

[3] Cheng Yanlin. Features of Broadcasting and Hosting in the Self-Media Era[J]. Sound Screen World: November 2017.

[4] Lou Shuhong. Self-media's shaping of the host's image [J]. Youth reporter: February 2016.

[5] Wu Wei. Talking about the types and characteristics of online program hosts News World: Issue 4, 2017. 
[6] Li Yue. The Pastime and Criticism of Online Talk Show Tucao Culture_-Taking "Rampaging Incident" as an example News Outpost Issue 11, 2015.

[7] Yi Yimeng. Talking about the network host Wang Nima's language art fits the audience's spirit Mass Literature and Art Issue 6, 2017.

[8] Liu Linxiao, Li Li. On the development of "host-centered system" in talk shows Liaoning Technical University June 10, 2014.
[9] Jia Ji. The Elements of Success for Chinese Talk Show Hosts China Radio and Television Journal, Issue 7, 2012.

[10] The construction and maintenance of virtual idol personification symbols from the perspective of media integration[J]. Li Yaxin. New Media Research. 2021(14).

[11] Semiotic interpretation of Vlog of professional life_-Taking bloggers of station B as an example [J]. Zhang Yuqi, Liu Yuxiao. Northern Media Research. 2021 (01). 